\title{
Memory and switching effects in polycarbonate-graphite composites
}

Cite as: Journal of Applied Physics 58, 1061 (1985); https://doi.org/10.1063/1.336217

Submitted: 08 November 1984 . Accepted: 12 March 1985 . Published Online: 14 August 1998

T. A. Ezquerra, F. J. Baltá-Calleja, D. R. Rueda, and J. Plans

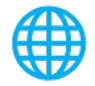

\section{Challenge us.}

What are your needs for periodic signal detection?

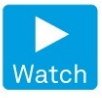

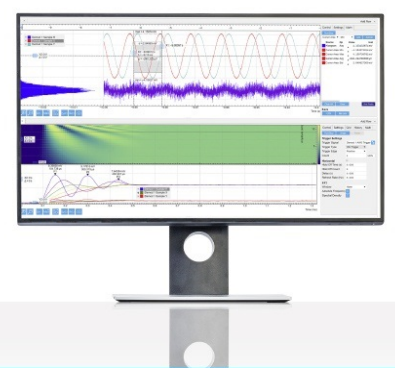

Zurich - Instruments 
T. A. Ezquerra, F. J. Baltá-Calleja, and D. R. Rueda

Instituto de Estructura de la Materia, C.S.I.C. Serrano, 119, 28006 Madrid, Spain

J. Plans

Departamento de Física Fundamental, Universidad Autónoma de Madrid, Cantoblanco, Madrid, Spain

(Received 8 November 1984; accepted for publication 12 March 1985)

Reproducible memory and switching effects have been detected in polycarbonate-graphite composites. Memory effects are characterized in the range of $0-1 \mathrm{kV} / \mathrm{cm}$. It is suggested that they involve electrothermal and electromechanical phenomena. Switching effects occur above $2 \mathrm{kV} /$ $\mathrm{cm}$ and are related to an intrinsic electric breakdown of the material. The threshold field increases with increasing thickness of the composite film. The temperature dependence and level of conductivity reached point toward a prevailing electron tunneling mechanism.

The increasing interest in polymeric composite materials partly derives from the fact that they offer alternative modes to develop light systems with conducting electrical properties. ${ }^{1,2}$ When a few percent conducting solid microadditive is mixed with an insulating polymer matrix, the overall conductivity increases towards a conducting level at a critical percolation volume of the microadditive. ${ }^{3}$ This effect has been used to produce materials with thermal switching possibilities. ${ }^{4}$ In a previous work we reported some preliminary results on polycarbonate filled with carbon black ${ }^{5}$ showing the development of an interesting class of light conductive material. The present study shows that when using graphite, instead of carbon black as a filler, a kind of electrical memory and current switching effects can be developed.

Several mixtures of polycarbonate PC (Bisphenol $A$, from Bayer) have been prepared using a plastograph with concentrations up to $30 \mathrm{wt} \%$ of natural graphite powder from SEA Tudor (mean grain size $\sim 15 \mu \mathrm{m}$ ). The original polycarbonate was first melted at a working temperature of $230^{\circ} \mathrm{C}$. Graphite was then added and the mixture was stirred until a homogeneous system was reached. Samples for electrical measurements have been prepared in the form of 300 $700-\mu \mathrm{m}$-thick films, by pressure-molding at $50 \mathrm{bar}$ and $230^{\circ} \mathrm{C}$ the resulting composite material of the plastograph. Electrodes of 3-5 mm in diameter were prepared by evaporation of Au-Pd onto the film surface. Electrical properties were measured across the film thickness using a Keitley potential source of $3000 \mathrm{~V}$. The voltage and the current were measured with a Data Precision voltmeter and a Leeds \& Northrup, current recorder, respectively.

When a dc field $E$ is applied to a fresh PC-graphite sample, the current density $J$ increases from an initial value $J_{0}$ to a limiting one. Qualitatively, a similar behavior has been recently reported in case of PVC-graphite films. ${ }^{6}$ Figure 1 shows the transient current density at different fields for a 30 wt. \% graphite sample. The lowest field applied was 184.2 $\mathrm{V} / \mathrm{cm}$. Each field immediately higher was only applied after a saturation current density was reached. The saturation current density value $J_{\text {sat }}$ for each $E$ could only be achieved several hours after the field was applied. The value of $J_{\text {sat }}$ (taken as $\Delta J / \Delta t<1 \%$ for $\Delta t \sim 1 \mathrm{~h}$ depends on the applied field value. For a low value $E=184.2 \mathrm{~V} / \mathrm{cm}$, one reaches $J_{\text {sat }}=0.5 \times 10^{-3} \mathrm{~A} \mathrm{~cm}^{-2}$ after $5 \mathrm{~h}$ while for $E=548.7 \mathrm{~V} /$ $\mathrm{cm}$ one needs more than $30 \mathrm{~h}$ before reaching
$J_{\text {sat }}=9.22 \times 10^{-3} \mathrm{~A} \mathrm{~cm}^{-2}$. After reaching an arbitrary highest field (in our case $911.1 \mathrm{~V} / \mathrm{cm}$ ), we now decrease $E$ following the same steps as in the upward trend. In this way more conductive $J$ values, for the same applied fields as before, are obtained. This is shown in the field-saturation current-density characteristics of Fig. 2. The system shows a conspicuous memory effect related to the above-described electrical history. The relaxation processes involved in these transient currents may be tentatively understood in terms of electromechanical microstructural effects. It has been previously shown that electromechanical stresses produced by the field applied across a dielectric induce a compressive strain within the dielectric material. ${ }^{7}$ According to the calculations of Blok and Le Grand 7 the application of an electric field will produce a reversible compression if the deformation is smaller than $40 \%$, the initial thickness (elastic limit). A further increase of the field will cause a compression of the dielectric which yields plastically if the intrinsic

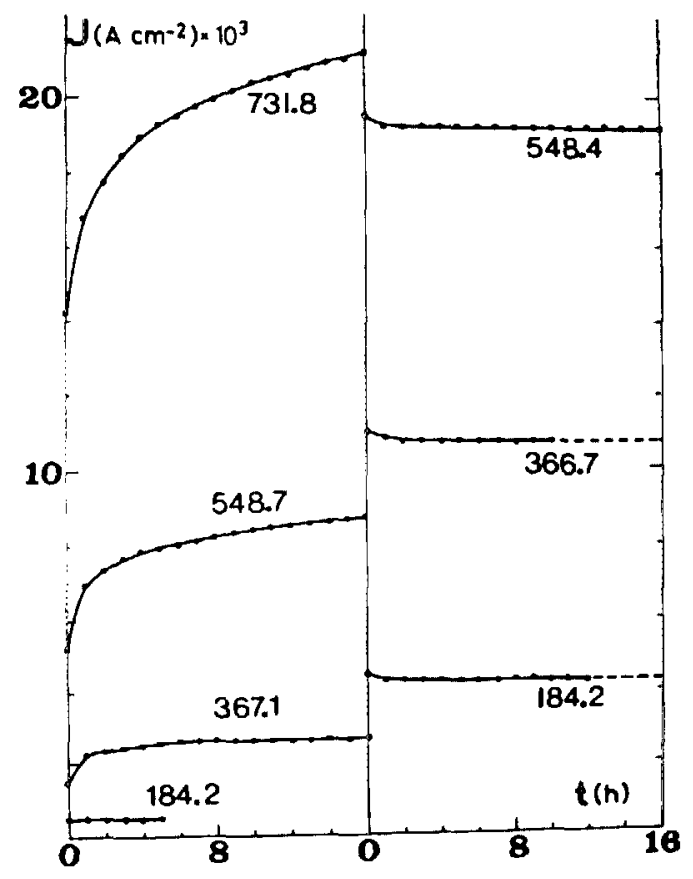

FIG. 1. Current density as a function of time at different applied fields for 30 wt. \% graphite-polycarbonate composite. Film thickness: $500 \mu \mathrm{m}$. $J$ values obtained after application of increasing fields $E(\mathrm{~V} / \mathrm{cm})$ (left) and after application of decreasing fields (right). 


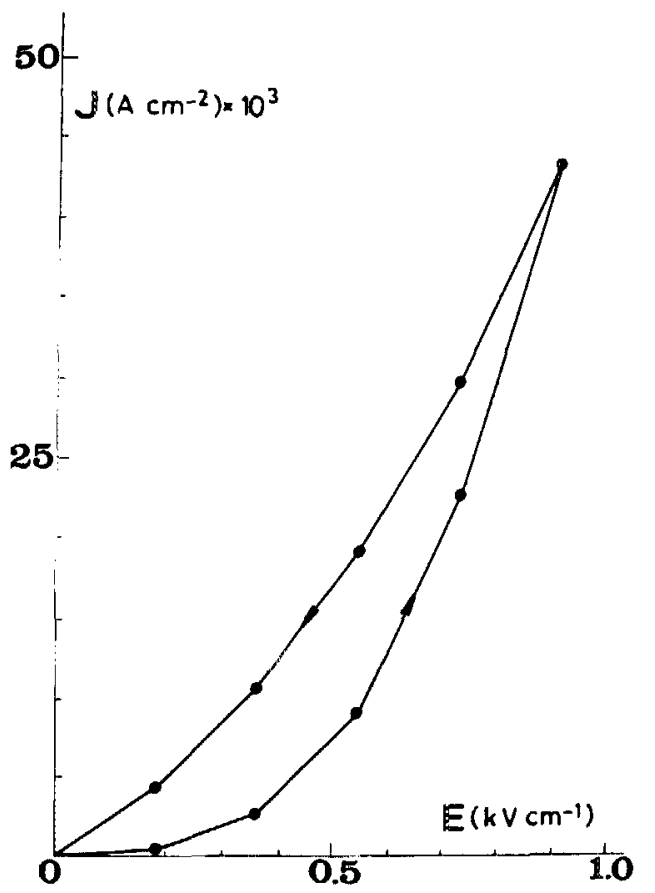

FIG. 2. Saturation current-density values for the composite sample of Fig. 1 as a function of applied field. The arrows denote the increasing trend of the applied field up to $E \sim 0.91 \mathrm{kV} / \mathrm{cm}$ (see text) and the higher data obtained when decreasing $E$ through the same field values.

breakdown of the dielectric material has not been reached. Hence the interparticle field may contribute to a lowering of the distance between neighboring particles favoring the current-density increase. It is noteworthy that average interparticle distances of the order of some nanometers would be required to plastically deform the polymer matrix. When the electric field is removed, a permanent distortion of the ma-

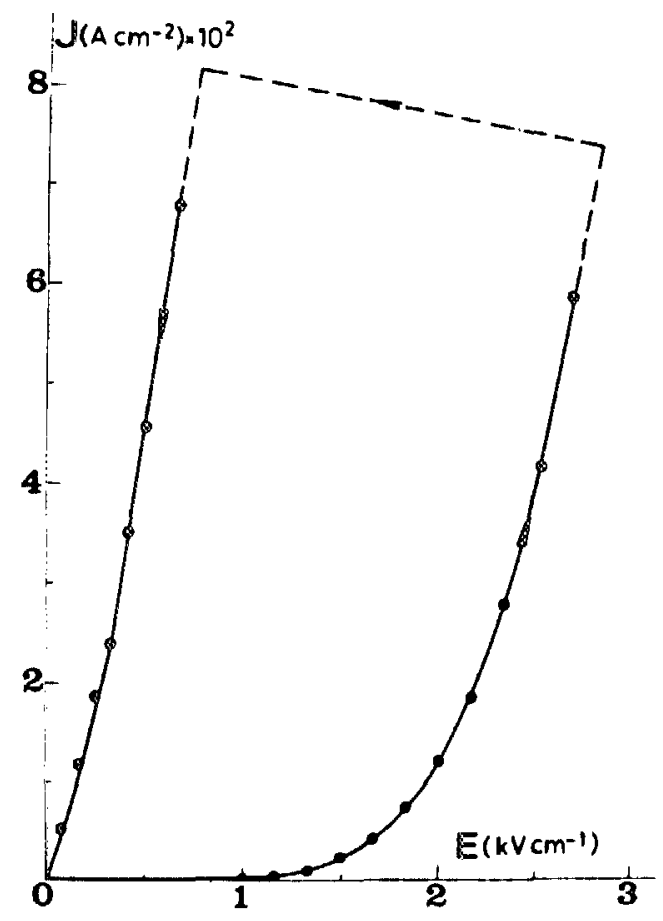

FIG. 3. Application of larger fields gives rise to a switching effect at a threshold field $E_{\mathrm{th}} \gtrsim 2 \mathrm{kV}$, which finally leads to a four orders of magnitude higher conductive material (specimen thickness: $600 \mu \mathrm{m}$ ).

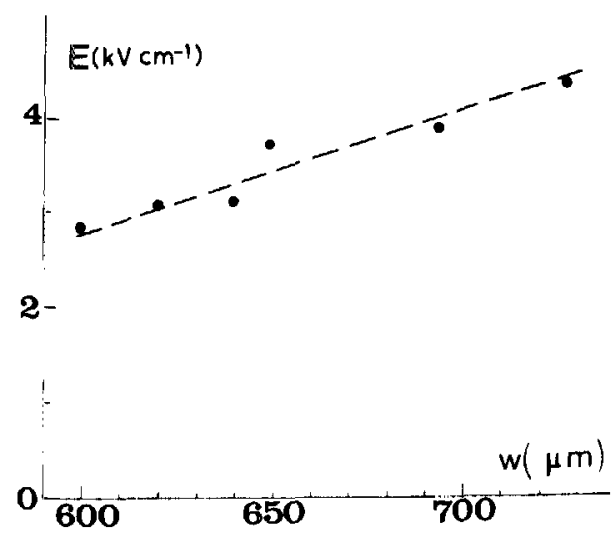

FIG. 4. Plot of threshold field as a function of specimen thickness.

trix would modify the average distance between particles giving rise to a more conductive state than the intial one. Analogous memory effects are obtained for lower graphite concentrations than $30 \mathrm{wt} . \%$.

If higher electric fields ( $>2 \mathrm{kV} / \mathrm{cm}$ ) are applied without letting the saturation state be reached, an effect like switching appears at a threshold field $E_{\mathrm{th}}$. This field $E_{\mathrm{th}}$ otherwise leads to higher $J$ values which are off the range of our present equipment. After reaching $E_{\mathrm{th}}$ an irreversible state, having a conductivity level four orders of magnitude higher, is obtained (Fig. 3). This process is not dependent on the polarity and involves a slight local modification of the surface of the sample. The threshold field increases linearly with the thickness of the film as shown in Fig. 4. These results suggest the occurrence of an electric breakdown causing interparticle discharges leading eventually to local melting of the matrix. Current-carrying tracks of graphite particles are formed along the discharge path, as we have seen in parallel micro. scope observations. Results of Fig. 4 qualitatively agree with recent data on switching effects in polymer thin films. ${ }^{8,9}$

Finally, Fig. 5 shows the temperature dependence of the electrical conductivity $\sigma$ for the polycarbonate composite samples having two different concentrations of graphite. Saturation current-density values were achieved before varying the temperature $T$. The decrease of $\sigma$ with $T$ shows that the system deviates from that corresponding to a pure graphite conductivity, ${ }^{10}$ suggesting that a conducting network is not yet completely developed. The conductivity level measured is sufficiently high $(\sim 10$ orders larger than that of the polycarbonate matrix) to ensure that the percola-

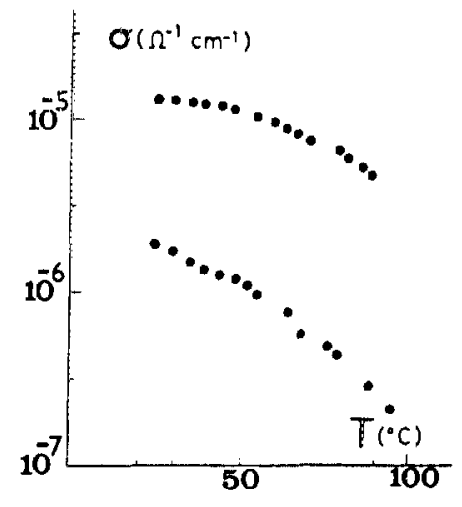

FIG. 5. Temperature dependence of the electrical conductivity for two polycarbonate-graphite composites having 20 and 30 wt. \% concentration of graphite (lower and upper, respectively). 
tion volume has been reached. Nevertheless, the level of $\sigma$ is still about 5-6 orders of magnitude lower than expected for graphite. Thus, one could invoke a conduction mechanism involving a double contribution leading to the intermediate levels of $\sigma$ observed. On the one hand, a limited physical contact between neighboring particles forming tracks accross the sample is expected. On the other hand, electron tunneling across bundles of grapite particles which are sufficiently close to each other would be required to account for the negative slope of the plot $\log \sigma$ vs $T$ (Fig. 5). The latter contribution seems to be the most dominant one.

Grateful acknowledgment is due to SEA Tudor, Madrid and to CAICYT, Spain for the generous support of this investigation. One of us (T.A.E.) wishes to thank the PFPI,
Ministry of Education and Science for the tenure of a research scholarship.

'D. M. Bigg and E. J. Bradbury, Conductive Polymers, edited by R. B. Seymour (Plenum, New York, 1981), p. 23.

${ }^{2}$ D. E. Davenport, in Conductive Polymers, edited by R. B. Seymour (Plenum, New York, 1981), p. 39.

${ }^{3}$ F. Bueche, J. Appl. Phys. 43, 4837 (1972).

${ }^{4}$ F. Bueche, J. Appl. Phys. 44, 532 (1973).

${ }^{\text {5F }}$. J. Baltà-Calleja, T. A. Ezquerra, D. R. Rueda, and J. Alonso-López, J. Mater. Sci. Lett. 3, 165 (1984).

${ }^{6}$ K. S. R. C. Murthy, K. Ramkumar, and M. Satyam, J. Mater. Sci. Lett. 31, 813 (1984).

'J. Blok and D. G. Le Grand, J. Appl. Phys. 40, 288 (1969).

${ }^{8}$ H. Carchano, R. Lacoste, and Y. Segui, Appl. Phys. Lett. 9, 414 (1971).

'Y. Sakai, Y. Sadaoka, and G. Okada, J. Mater. Sci. 19, 1333 (1984).

${ }^{10}$ W. W. Tyler and A. C. Wilson, Jr, Phys. Rev. 89, 870 (1953). 